after the acute illness has subsided, and that it does not remain for months or even years afterwards, as is so often incorrectly taught. Our own experience is in accordance with the views of these observers.

\section{A CEREBRO-SPINAL MANOMETER. ${ }^{1}$}

By FRaNK C. EVE, M.D. CantaB., M.R.C.P.Lond., ASSISTANT PHYSICIAN, VICTORIA HOSPITAL FOR CHILDREN, HULL; LATE HOUSE PHYSICIAN, HULI ROYAL INFIRMARY.

I was led to devise this simple instrument for measuring the cerebro-spinal pressure by the freedom with which many authors have invoked an excess of intracranial tension as the explanation of various clinical phenomena without any measurement of that pressure.

The following is a description of the instrument with an abstract of as much of the results of about 50 clinical cases as space will allow. It will be seen that the instrument is easy and safe in application and that the diagnostic and therapeutic results have of ten been surprisingly good. I had believed myself first in the field, but I find that Professor Krönig of Berlin has invented a more complicated instrument for the same purpose.

The instrument consists of the lumbar puncture needle, which is adapted on the one hand to a vertical graduated glass tube and on the other to an exit tube closed by a spring clip. There is also a handle which is detachable. The three hollow needles supplied are suitable for children, large or small adults. These needles are all tempered as tough as possible, so that the risk of their breaking in situ is avoided. Included in the case is a hydrometer for measuring the specific gravity of small quantities of the cerebro-spinal fluid. Just before use the whole apparatus is boiled and brought to the bedside in a dish of boiled water. The patient lies on his side with the knees drawn up and the back arched. The head and spinal column must be approximately horizontal. In uncontrollable patients it is
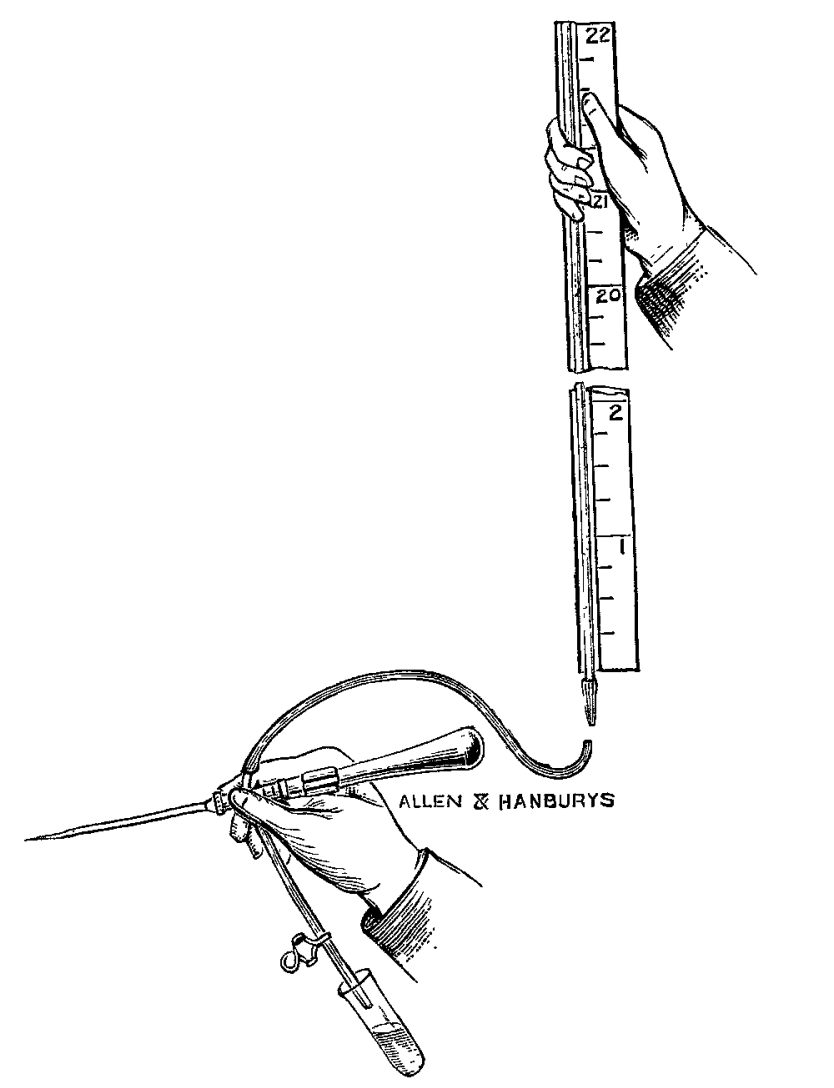

very helpful to pass a long strap beneath the knees and round the shoulders. This arches the spine and renders struggling quite futile. In nervous patients a little cocaine beneath the skin and a little more near the bone is recommended but the pain is not great, being about equal to that of aspiration of the chest.

The skin is carefully cleansed and the needle is introduced at the level of the iliac crests half an inch below and external to a vertebral spine. The spinal cord terminates

1 Made by Messrs. Allen and Hanburys, Limited, 48, Wigmore-street, several inches above this point. The needle is pushed in with a slightly upward tilt. If bone is struck the direction must be slightly altered until the firm soft resistance of the ligamentum flavum is encountered. Once past this no further resistance is felt and the point of the needle finds itself free in the lake of cerebro-spinal fluid amongst the cauda equina. Cerebro-spinal fluid now runs up the manometer tubing (the exit tube having been previously clamped) and its pressure can be read off. Sometimes the cerebro-spinal fluid requires a little coaxing, by milking or pinching the rubber tube before it will flow into the manometer. Forcible aspiration, as by a syringe, should never be employed. Fluid can now be allowed to trickle from the exit tube by removing its clamp and clamping the manometer tube. The needle is withdrawn when the pressure is sufficiently reduced or the requisite amount of fluid has trickled out. An ounce or an ounce and a half is frequently withdrawn and usually leaves the pressure about zero. The fluid is set aside to see if it clots and is examined by the hydrometer, the microscope, or other means.

In children the operation is easy but in an average man the needle has to traverse three inches of flesh, so that one must be prepared for a little patient probing in difficult cases. Rigid asepsis is of course imperative but I have never seen any septic troubles and the fluid will keep in a test tube a surprisingly long time without obvious decomposition. The apparatus shows clearly the oscillations in cerebrospinal pressure produced by the pulse and respirations. In fact, one has the whole central nervous system in a perfect natural bony plethysmograph.

The first 24 cases were all described to the Hull branch of the British Medical Association. Space now only allows a selection of the more noteworthy cases.

CASE 1.- The patient was a boy, aged 13 years, who three weeks previously had been knocked on the head with a bat. For a week he lay in the hospital in a drowsy, apathetic condition, with headache, facial twitchings, and occasional vomiting. The morning after half an ounce of cerebrospinal fluid had been withdrawn he was transformed into a bright and cheerful boy. His pressure was ten inches, which is higher than normal (from three to eight inches).

CASE 2. Pontine hemorrhage.-The patient was moribund. Lumbar puncture was accidentally delayed, so that it was only completed when the last few respirations were being taken. The pulse continued a good deal later. The fluid was pink and the pressure was eight and a half inches. Three drachms were withdrawn, reducing the pressure to five inches. This pressure was sustained after death with no sign of diminution after several minutes. I attribute this to the engorged condition of the venous system. The pink fluid was a valuable diagnostic guide to the apoplectic origin of the coma; this was verified post mortem.

CASE 3. Concussion. - The patient was a boy, aged eight years. The case was diagnosed as fractured base ; the fluid, however, was colourless and the pressure was only six and a half inches, which corrected the diagnosis into concussion. He was much better after the puncture and recovered.

CASE 5. Fractured base (folloned by meningitis). - The patient was a man, aged 54 years; he was bleeding from the ear and nose. Lumbar puncture on the eleventh day gare a pressure of seven inches only but the fluid was slightly turbid and clotted on standing. This gave a diagnosis of meningitis which was only a suspicion otherwise and which was confirmed by subsequent symptoms and by post-mortem examination. On the thirteenth day the pressure appeared to rise quickly from two and a half to ten inches, owing to fright from a little pool of burning ether which was accidentally lighted near the patient's nose. He was semi. conscious at the time.

CASE 10. Certbral thrombosis.-The patient was a man, aged 57 years, who for a week had been developing left hemiparesis with severe headache. Three days later he was comatose and seemed to be dying, with a slow feeble pulse. With strychnine and two lumbar punctures (three dracbms each) he soon recovered consciousness and could talk in three weeks and walk in two months. His recovery seem erl remarkable.

CASE 11. Pneumonia.-CThe patient was a man, aged $=0$ years, with intense toxæmia and violent delirium. Orse ounce of clear fluid was withdrawn, reducing the high pressure of from 12 to 18 inches down to three inches. No improvement followed. The $\mathrm{ligh}$ pressure in this case was doubtless due to the distended right heart and veins.

CASE 14. Fractured vault.-The patient was a child, agud 
five years. As the clot was extra-dural the cerebro-spinal fluid was not blood-stained. No benefit ensued.

CASE 15. Acute tuberculous meningitis. - The patient was a child, aged one year. Lumbar puncture was performed for vomiting and convalsions. The pressure was 13 inches and the fluid was clear. There was very marked improvement for two days. Puncture was repeated on the sixth day when the pressure had again risen to 12 inches. Improvement was now very transient. Diagnosis was verified post mortem. 'This was my first measurernent of cerebro-spinal pressure (February, 1903).

CASE 16. Chronic hydrocephalus.--No fluid was obtainable in this case (a child, aged three years), the cranial cavity evidently being shut off.

CASE 17. Cerebral tumour.-The patient was a man, aged 45 years. There was a history of pains in the back of the head and neck for three months, together with vomiting and early optic neuritis. Here again, lumbar puncture yielded only a few drops of clear fluid. The pressure was not measureable even on coughing or sitting upright. The probable explanation of this is a sub-tentorial tumour pressing down the cervical medulla and cerebellum into the funnel-shaped foramen magnum. If this be the true explanation lumbar puncture gives a new and useful localising sign in this case. (Since the above was written the necropsy has shown a tumour, as large as a pigeon's egg, in the right lobe of the cerebellum.)

CASE 20. Pontine apoplexy.-The patient was a man, aged 45 years. Here again no fluid could be obtained except a few drops at a pressure of nine inches. Postmortem examination showed a clot in the pons as large as a walnut which had ciearly corked off the cerebral from the spinal cavity. Expansion upwards was prevented by the strong tentorium cerebelli.

CASE 21. Double optic neuritis. - The patient was a healthy boy, aged ten years. A history of one month's failing vision was obtained without any assignable cause. There were practically no headackes or vomiting. The cerebrospinal pressure was very high, exceeding 16 inches, which was then the limit of the manometer. After one and half drachms had been withdrawn it was still 15 inches. The boy was thoroughly at his ease during the process so I tried the effect of mental arithmetic. This first increased the pressure about an inch and then lowered it one and a half inches; grasping with both hands raised the pressure two and a half inches. As usual in a high-pressure case, the oscillation of the pulse was well marked and the respiratory wave was scarcely seen. 11 drachms of fluid were withdrawn, reducing the pressure to four and a half inches. The puncture was repeated in ten days when the pressure was two inches and only half a drachm of fluid could be withdrawn. The punctures appeared to have no effect on the steady downward progress of his vision. He vomited six and 18 hours after the puncture and had a trifling headache. Nine months later the father told me that the boy was well and cheerful but had nocturnal incontinence and slightly impaired memory. He could see a man 200 yards away and his vision was better after an aperient. This case suggests the possibility of disease of cerebro-spinal high tension analogous to glancoma in the ocular cavity.

CASE 22. Fractured base.-The patient was a man, aged 76 years. It was a severe and undoubted case. The pressure was four and a quarter inches. The fluid was pink. Recovery was complete.

CASE 23. Fractured base.-The patient, aged 19 years, was comatose. The pressure was from 10 to 13 inches and was reduced to five by withdrawing one and a half ounces of pink fluid, after which the pulse was less turbulent. Recovery was rapid and complete.

CASE 24. Severe chorea.-The patient was aged 22 years. There was decided benefit from the puncture. The pressure was normal.

CASE 25. Concussion.-The patient was aged eight years. Tomiting, teeth-grinding, twitching, and speechlessness were present. Complete recovery followed a few hours after puncture (one drachm) The pressure was normal.

CASE 26. Tuberculous meningitis.-The pressure was $19 \frac{1}{2}$ inches. The child slept well after puncture-a common result. Improvement was transient.

CASE 27. Uremic convulsions with total blindness.-The patient was a middle-aged man. The convulsions were relieved by venesection but the coma remained. Lumbar puncture under chloroform was performed by Dr. Carter. The pressure was high ( $16 \frac{1}{2}$ inches). On the next morning the man was quite conscious and had recovered much of his eyesight. Dr. W. C. Rockliffe, our ophthalmic surgeon, examined the eyes and found very marked optic neuritis. He was much impressed by the extent and rapiclity of the recovery of vision. A second puncture produced no further increase of vision.

CASE 28. Anomalous cerebellar ataxia.-The patient was a man, aged 35 years, with a history of pain, numbness, and feeble gait for six years, and a strong family history of adolescent paralysis. The diagnosis was obscure. Extraordinary improvement followed after withdrawing one onnce of fluid at normal pressure, suggesting functional disease. He was at work six months later, quite well.

Case 29. Acute tuberculous meningitis.-C'The patient was a child, aged seven years, who three months previously I had seen operated on for undoubted tuberculous peritonitis. The child was admitted for acute periostitis of the ankle. No pus was found on incision. Five days later the child had a severe clonic convulsion lasting for two hours, preceded by several days of irritability and sulkiness. The fit ceased 15 minutes after lumbar puncture, which showed a pressure of $15 \frac{1}{2}$ inches. Four days later, vision, which apparently had been totally lost since about the time of the fit, was returning. Five months later the mother tells me the child's health is normal in every respect. I have to thank Mr. G. A. Carter for a careful report of this striking case which occurred in my absence.

Negative cases. - The cases where Iumbar puncture produced little or no result included Ménière's disease (severe and senile case), alcoholic neuritis and myelitis, neurasthenia, miliary tuberculosis, chronic headache, and gastrio tabetic crises. The amount of fluid withdrawn was usually between half an ounce and one and a half ounces and the pressure varied between zero and 18 inches of water.

Conclusions.-The following points appear to be suggested by the above cases :-

1. The therapeutic effect of lumbar puncture is, I think, often greater than it is given credit for. This is difficult to prove or to explain (and hence to believe) but the following may be factors: (a) excessive tension is reduced; $(b)$ the brain is washed by fresh fluid secreted in place of that withdrawn ; (c) a temporary cerebral hyperæmia is produced by the blood-vessels dilating to take the room of the fluid withdrawn until a new supply is secreted, or, conversely, a cerebral anzemia due to excessive intracranial pressure may be arrested; and (d) a substantial change of cerebral environment is produced of which nature may sometimes be able to take advantage.

2. Lumbar puncture has been performed about 60 times with this instrument without any ill-effects, so that the risk is not great, and the further exploration of this therapeutic field is justified.

3. Valuable diagnostic information is often afforded. (a) Pink fluid indicates cerebral hæmorrhage, fractured skull (with injury to the dura mater), or severe superficial cerebral laceration (with rupture of the pia mater). Except for the first few drops the fluid is very seldom blood-stained from trauma by the needle with this instrument. (b) A faint "spider's web" clot after standing several hours points strongly to meningitis. (c) Cytological, bacteriological, or inoculation experiments may give the diagnosis. (d) Occlusion of the cerebral from the spinal cavities may be diagnosed (see Cases 16, 17, 20) and this may localise a subtentorial tumour (Case 17).

4. In apoplexy, lumbar puncture is conceivably justifiable when the pressure is found to be very high, provided that the pressure is slowly reduced to half its value, not too soon after the occurrence of the hæmorrhage. Baiv if this treatment should be found to be justifiable with a manometer, it would certainly be dangerous without one.

5. Case 12 suggests the possible existence of a disease in the cerebro-spinal cavity analogous to glaucoma in the ocular cavity - that is, a disease of high pressure due to obstructed outflow of secretion. There are many cases of neuritis and atrophy of the optic nerve which still require an explanation.

6. Lumbar puncture is said to be dangerous in cerebral tumour. The sitting-up position advised by some French and German authorities has serious theoretical risks.

7. Credit for therapeutic effects is hard to allocate, but surprisingly good results followed lumbar puncture in fractured base (Cases 22, 23), concussion (Cases 3, 25), posttraumatic cerebral condition (Case 1), cerebral thrombosis (Case 10), very severe chorea (Case 24), uramic coma and 
optic neuritis (Case 2), anomalous ataxia case (Case 28), and tuberculous meningitis (very early stage) (Case 29).

8. Hence, I think that lumbar puncture should be tried as a therapeutic effort in a variety of cerebral conditions which are not hopeless but which are stationary or retrocedent under ordinary treatment. Also that the cerebro-spinal pressure should be measured $n$ every case of lumbar puncture for the present.

This investigation would have been impossible without the kindness of the honorary and resident staff of the Hull Royal Infirmary. $\mathbf{M y}$ sincere thanks are therefore due to Dr. G. F. Elliott, Dr. Frank Nicholson, Dr. E. O. Daly, Mr. E. H. Howlett, Dr. D. Lowsor, Dr. Rockliffe, Mr. E. Harrison, Mr. A. G. Francis, Mr. Guy B. Nicholson, and Mr. Carter ; also to Sir Victor Horsley.

Hull.

\section{atiledical Soctieties.}

\section{CLINICAL SOCIETY OF LONDON.}

Malarial Affection of a Joint.-Intermittent Hydrops of the Joints.-Hydronephrosis due to Moveable Kidney.

A MEETING of this society was held on April 14th, Dr. FREDERICK TAYLOR, the President, being in the chair.

Professor HowaRD MARsH communicated a case of Affection of a Joint possibly due to Malaria. The patient was a man, aged 38 years, in whom there was a history of syphilis and who had lived abroad and contracted malaria. In April, 1903, his knee became the seat of severe pain but without swelling or loss of movement. When first seen in December, 1903 , the joint appeared natural but the muscles were very much wasted and were so weak that he was unable to raise his heel from the bed. He complained of agonising pain from his hip to his ankle. Every evening the joint became considerably swollen and measured one and a half inches more than the other, the swelling having a remarkably globular appearance. There was no fluid in the joint and the swelling seemed due to vascular turgescence of the synovial membrane. The patient stated that the joint swelled up in this manner every evening and then gradually returned to its natural size. After the administration of quinine in ten-grain doses twice a day for four days the attacks of swelling had disappeared and the patient recovered muscular power in the limb so rapidly that in three weeks he was able to walk three or four miles without any bad result. Six months later he had a patch of swelling covered by overwarm and hyperæsthetic skin just over the internal malleolus, worse at night, which was similarly relieved by quinine. The blood was examined by Major Ronald Ross and no parasites were found, but on account of the periodicity of the swelling of the knee recurring at intervals of 24 hours, and that it was attended with severe neuralgic pain and relieved by quinine, Professor Marsh believed it was possible that these symptoms were due to malaria and referred to the "brow ague," neuralgiæ, and other symptoms occurring in malarial subjects.-Sir PATRICK MANSON remarked that Professor Marsh's case had again raised a question which had been often discussed but never settled-namely, the position of certain irregular manifestations of malaria. There were two ways in which these manifestations might be related to malaria-first, as a direct symptom of the disease and, secondly, as a condition due to the lowered vitality caused by the malaria. Thus syphilitic or other poisons produced a greater effect in a person who had had malaria than in a healthy person. In Professor Marsh's case he could see no reason for supposing that the condition of the joint was due to malaria, as the patient presented no symptoms which were pathognomonic of that disease. The periodicity was quotidian, a periodicity which was common to many conditions and was usual in all septic fevers. In malaria the periodicity would be typically tertian or quartan. Moreover, in this case the exacerbation of symptoms had been in the evening, which was a common event in syphilis but rare in malaria; the typical feature of malarial symptoms was their occurrence before midday. There were no other manifestations of malaria, such as fever and enlargement of the spleen. He paid less attention to the absence of the malarial parasite from the blood, for quinine had been previously administered; it would have been more useful to examine the blood before this administration. He would like to know how recently the patient had been exposed to malarial infection, for in his experience no active evidences of malaria ever occurred after three years at the utmost from such possible infection. He referred to various conditions that had been termed irregular malaria, notably periodic nervous, cutaneous, and gastric symptoms. He did not know of any that had harl the two great malarial test applied to them-namely, finding of the parasite and observance of the malarial periodicity, though he referred to two cases of urticaria which were probably malarial on account of their clinical features. The malarial parasite undoubtedly had a predilection for certain organs but he doubted if the joints should be included among them.Lieutenant-Colonel T. R. MULRonEY, I.M.S., had seen many cases of malaria complicated by syphilis, the manifestations of which were more decided than in non-malarious subjects and were often temporarily relieved by quinine. But iodide cf potassium was necessary to cure them permanently as in a case which he quoted of affection of the ankle-joint. Professor MARSH replied and stated that over three years had elapsed since the man had been abroad.

Professor MARSH also made a communication on three cases of Intermittent Hydrops of the Joints occurring in a woman aged 28 years, a man aged 42 years, and a boy aged 12 rears. These three cases were in all their essential features very similar to each other. The effusion in the joints returned with remarkable periodicity : in two every 14 days and in one every 12 days. In all the local condition-mere increase of synovial fluid-was the same. In all, the joints in the intervals seemed normal. These cases were typical instances of the usual form of intermittent hydrops of the joints and they closely resembled those which were observed or recorded by Dr. E. J. Brackett and Dr. F. I. Cotton. In these examples the periodicity was always marked lut the interval between the attacks varied in different individuals from three or four days to 30 days or more, the most frequent interval being 14 days. In several instances the attacks continued for three, four, or even more years. Some cases after showing definite periodicity lost this feature and the attacks became irregular both as to the time of their recurrence and their severity. The pathology of this affection was not known. It might be that under the influence of some cause not yet recognised a vaso-motor disturbance led to synovial effusion of a periodic character. In regard to treatment, arsenic was the only agent which appeared to be useful. These cases were not only interesting on their own account but because they might lead to errors in practice, for the recurring effusion might very well suggest the presence of a loose cartilage or a synovial fringe for which a useless operation might be performed. -Mr. F. C. WALLIS expressed the belief that snch cases as those now described by Professor Marsh were due to some micro-organism or its toxin and thought it would be interesting to know the results of a series of cultures taken from the fluid in the joint. He had opened and washed out the affected joint in one case with advantage.-Dr. F. J. POYNTON pointed out that micro-organisms in affections of the joints were commonly confined to the subendothelial layer and were rarely found in the fluid. He was not of opinion that the cases described were of microbic origin but rather of the nature of an cedema or an urticaria. It was, however, well known that the subcutaneous injection of certain toxic and antitoxic sera, such as those of diphtheria and tetanus, could produce synovial effusion. Had urticarial or erythematous lesions been observed in Professor Marsh's cases.-Dr. F. W. ForBas-Ross thought that the effusion was probably a serous hæmorrhage into the joint, due to a diminution in the coagulability of the blood. Malaria was known to predispose to this condition and oderna often occurred in such subjects after the administration of mercury.-The PREsident asked on what grounds arsenic bad been given.-Professor MARsH said that those who had seen many of these cases recommended arsenic but some cases did not improve with it, whereas most cases spontaneously improved. He did not think that the condition was of infective origin, for there was no structural alteration of the joints, even after repeated attacks. He had not examined the synovial fluid or the blood in his cases. Dr. A. E. Garrod had told him of three cases which he had seen, two of which were the direct sequelæ of gonorrhœal rheumatism.

Mr. F. J. STEWARD communicated two cases of Hydronephrosis due to Moveable Kidney. These two cases were 